\title{
Evaluating the phase diagram of superconductors with asymmetric spin populations
}

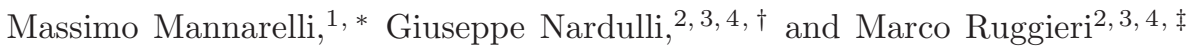 \\ ${ }^{1}$ Center for Theoretical Physics, Massachusetts Institute of Technology, Cambridge, MA 02139 \\ ${ }^{2}$ Università di Bari, I-70126 Bari, Italia \\ ${ }^{3}$ I.N.F.N., Sezione di Bari, I-70126 Bari, Italia \\ ${ }^{4}$ TIRES, Centre of Innovative Technologies for Signal Detection and Processing, University of Bari, I-70126 Bari, Italia
}

(Dated: October 13, 2018)

\begin{abstract}
The phase diagram of a non-relativistic fermionic system with imbalanced state populations interacting via a short-range S-wave attractive interaction is analyzed in the mean field approximation. We determine the energetically favored state for different values of the mismatch between the two Fermi spheres in the weak and strong coupling regime considering both homogeneous and non-homogeneous superconductive states. We find that the homogeneous superconductive phase persists for values of the population imbalance that increase with increasing coupling strength. In the strong coupling regime and for large population differences the energetically stable homogeneous phase is characterized by one gapless mode. We also find that the inhomogeneous superconductive phase characterized by the condensate $\Delta(\mathbf{x}) \sim \Delta \exp (i \mathbf{q} \cdot \mathbf{x})$ is energetically favored in a range of values of the chemical potential mismatch that shrinks to zero in the strong coupling regime.
\end{abstract}

PACS numbers: 03.75.Ss,11.15.Ex

\section{INTRODUCTION}

Fermionic superfluidity driven by S-wave short range interaction is a collective phenomenon characterized by formation of correlated pairs of half-integer spin particles. This effect is relevant in many theoretical and experimental fields. Of particular interest are systems of trapped cold atoms of two fermionic species where the interaction between the fermions can be varied over a wide range employing a Feshbach resonance [1]. A general feature of such systems is that when the interaction between the fermions is increased the systems evolve from a weak-coupled superconductive BCS state of Cooper pairs to a Bose-Einstein condensate (BEC) of tightly-bound diatomic molecules [2, 3].

In a standard BCS superconductor the chemical potentials of the two fermionic species are equal. A small chemical potential difference $\delta \mu$ cannot disrupt BCS superconductivity. As a matter of fact the superconductive state with equal number densities is energetically favored in comparison with a normal state with a fermionic imbalance. On the other hand, as pointed out in [4] in the weak coupling regime, BCS superconductivity cannot persist for large values of $\delta \mu$. Indeed there exists an upper limit for $\delta \mu$ (the so-called Clogston limit), beyond which the homogeneous superconductive state is no longer energetically favored.

Systems with unequal populations of two fermionic species have been widely studied [4, 5, 6, 7, 8, 9, 10, 11, 12, 13, 14, 15, 16, 17, 18, 19, 20, 21, 22. For values of $\delta \mu$ larger than the Clogston limit several non trivial phases have been proposed. They include the Phase Separation (PS) state [8], the Breached Pair (BP) superfluidity [9, 10, 11], the Deformed Fermi sea pairing (DFSP) 7] and the non-homogeneous LOFF [6] phase (see [23] for a review).

Recent experiments with trapped cold atoms [24, 25] indicate that both the BCS and the BEC states can sustain large mismatches between the fermionic populations before turning to the normal unpaired state. For a wide range of values of such a mismatch it turns out that the superfluid atoms tend to remain in the inner core of the trap, whereas the normal atoms in excess are expelled and form a shell surrounding the center of the trap. These observations seem to support the PS scenario with phase separation [8] between superfluid and normal atoms. However, a clear signature of the PS phase is still lacking 24, 26].

The LOFF phase has not yet been observed in experiments with trapped cold atoms [24, 25], although it was seen in $\mathrm{CeCoIn}_{5}[27,28]$. The portion of parameter space where this phase is favored depends on the form of the condensate. In the simplest case, in the weak coupling limit this region is rather narrow and its detection in cold atom experiments might be difficult 24]. However a definite conclusion can be reached only after a detailed study of the space dependence of the condensate and the knowledge of its dependence on the coupling strength.

\footnotetext{
*Electronic address: massimo@lns.mit.edu

${ }^{\dagger}$ Electronic address: giuseppe.nardulli@ba.infn.it

‡Electronic address: marco.ruggieri@ba.infn.it
} 
The present study represents a preliminary analysis of this problem. We determine, in the mean field approximation, the phase diagram of an interacting two species fermion system as a function of the strength of the interaction and of their chemical potential difference, paying special attention to the LOFF region. We choose a four-fermion coupling to mimic the interaction, neglecting all the effects of the trap in the fermionic Hamiltonian [33]. Due to these limitations we do not attempt to make any comparison with experimental data, leaving this task to future studies.

Other analyses have been presented already on the same subject [14, 15, 16]. In particular in Ref. 14] a similar study has been performed for the homogeneous case. We differ from these authors for two reasons. First, we also include and discuss the LOFF phase. Second, we work at fixed total density but do not fix the difference in population between the two species. Under this respect our study may be seen as complementary to Ref. 14]. Due to these assumptions, our phase diagram at $T=0$ is given in terms of two parameters, $\delta \mu$ and the coupling constant, similarly to the analysis presented in [15].

One of our results is that the homogeneous phase is energetically favored in a range of values of the chemical potential difference that increases with increasing coupling strength. Moreover it turns out that the phase transition between the homogeneous and the LOFF phase is of the first order (this result was already known in the weak coupling regime and we extend it to the intermediate coupling regime) and there exists a critical value of the coupling constant where the LOFF window shrinks to a point. For larger values of the coupling the LOFF phase cannot be realized and the homogeneous phase has a first order phase transition directly to the normal phase. Further increasing the coupling strength and for large separations of the Fermi spheres the excitation spectrum of the energetically stable homogeneous phase is characterized by one gapless mode and the transition between the superconductive phase and the normal phase becomes of the second order.

As for the non-homogeneous phase we restrict our analysis to the condensate characterized by a one-wave oscillation $\Delta(\mathbf{x}) \sim \Delta \exp (i \mathbf{q} \cdot \mathbf{x})$. Non-homogeneous condensates characterized by a more complicated space dependence, such as that obtained by a superposition of several plane waves are expected to produce deeper minima of the free-energy $F[29]$. Therefore our study only provides a lower bound for the gap and the window in $\delta \mu$ where the LOFF state is energetically favored.

The stability of the homogeneous phase with different Fermi surfaces that we present in this article has been already discussed in Ref. 14]. We will only consider the solutions of our system of equations which are stable and that correspond to global minima of the free-energy. We do not examine the stability of the LOFF phase which however is expected to be stable [30].

Our paper is organized as follows. In Section $\amalg$ we study the homogeneous superconductive phase constrained by the conservation of the total number of fermions. In Section [II we discuss a LOFF phase characterized by a single plane-wave and we determine the width of the LOFF window as a function of the coupling. In Section [IV we summarize our results.

\section{MODEL AND HOMOGENEOUS PHASES}

We shall analyze a system comprising fermions of two species, with mass $m$, interacting via a short range (S-wave) four fermion interaction. The Hamiltonian density is

$$
\mathcal{H}=\sum_{\sigma=1,2} \bar{\psi}_{\sigma}(x)\left(-\frac{\nabla^{2}}{2 m}-\mu_{\sigma}\right) \psi_{\sigma}(x)-G \bar{\psi}_{1}(x) \bar{\psi}_{2}(x) \psi_{2}(x) \psi_{1}(x),
$$

where $G>0$ is the four fermion coupling constant. The chemical potentials of the two species can be written as $\mu_{1}=\mu+\delta \mu$ and $\mu_{2}=\mu-\delta \mu$, so that $\mu$ is the average of the two chemical potentials and $2 \delta \mu$ their difference. We restrict our analysis to zero temperature and work in the mean field approximation.

The effect of the attractive interaction between fermions is to produce a gap $\Delta$ in the quasiparticle dispersion laws which is related to the $\psi \psi$ condensate by

$$
<\psi_{\alpha}(x) \psi_{\beta}(x)>=\frac{\Delta(x)}{G} \epsilon_{\alpha \beta} .
$$

The non-homogenous superconductive phase, where the gap is not uniform in space, will be treated in the next Section. Here we only discuss the homogeneous case, $\Delta(x)=\Delta=$ const. In this case the excitation spectrum is described by the quasiparticle dispersion laws

$$
\epsilon_{1}=+\delta \mu+E_{p}, \quad \epsilon_{2}=-\delta \mu+E_{p},
$$

with

$$
E_{p}=\sqrt{\xi^{2}+\Delta^{2}}, \quad \xi=p^{2} / 2 m-\mu .
$$


Using the dispersion laws of the system one evaluates the grand-potential, which is given, at $T=0$, by

$$
\Omega=\frac{\Delta^{2}}{G}-\frac{1}{2} \int \frac{d^{3} p}{(2 \pi)^{3}}\left[\left|\epsilon_{1}\right|+\left|\epsilon_{2}\right|-2 \xi\right] .
$$

The integral in this expression is ultraviolet divergent and can be regularized by the usual procedure [3] employing the S-wave scattering length $a$ :

$$
\frac{m}{4 \pi a}=-\frac{1}{G}+m \int \frac{d^{3} p}{(2 \pi)^{3}} \frac{1}{p^{2}}
$$

We introduce the dimensionless coupling constant

$$
g=\frac{1}{\pi k_{F} a},
$$

where $k_{F}$ is the Fermi momentum. The weak coupling regime, where the BCS approximation holds, corresponds to $g \rightarrow-\infty$. This approximation is generally very good for superconductivity in metals. On the other hand, in cold atoms the strength of the interaction can be varied working in the vicinity of a Feshbach resonance, where the scattering length strongly depends on the applied magnetic field. Therefore both the weak and strong coupling regimes can be reached in this case.

The gap parameter $\Delta$ and the mean chemical potential $\mu$ can be determined self-consistently employing the equations

$$
\begin{aligned}
& \frac{\partial \Omega}{\partial \Delta}=0, \\
& \frac{\partial \Omega}{\partial \mu}=-n,
\end{aligned}
$$

where $n=k_{F}^{3} / 3 \pi^{2}$ is the fermionic number density. Eqs. (8) and (9) are the gap equation and the number equation respectively. In the weak coupling regime the chemical potential $\mu$ differs from the Fermi energy $\epsilon_{F}$ by an amount of order $\Delta^{2} / \mu^{2}$; therefore the approximation $\mu \simeq \epsilon_{F}$ is usually adopted and the number equation is not used.

Let us note explicitly that we do not write equations for $\mu_{1}$ and $\mu_{2}$ separately. We work at fixed $n$, but do not impose conditions on $\delta n=n_{1}-n_{2}$. As a consequence, we do not write down a third equation: $\frac{\partial \Omega}{\partial \delta \mu}=-\delta n$, which would be needed in the analysis if $\delta n$ were held fixed [14. Since $\delta n$ is not fixed, conversions between particles of different species are allowed and the thermodynamic potential whose minimum defines the vacuum state is not $\Omega$, but $F=\Omega+\mu n$.

\section{A. Numerical solution of the self-consistent equations}

In order to determine the actual ground state of the system we solve self-consistently Equations (8) and (9) to determine the values of $\Delta$ and $\mu$ for various values of the coupling $g$ and polarization $\delta \mu$. From the computed $\Delta$ and $\mu$ we evaluate the free-energy $F=\Omega+\mu N$ in the superconductive phase which we compare with the corresponding result in the normal unpaired phase. For $\delta \mu=0$ this analysis was performed in Ref. [31] and, as a test of our numerical code, we have reproduced all the results of this paper. In particular we mention some results valid at $\delta \mu=0$. The value of the gap is an increasing function of $g$. The average chemical potential $\mu$ decreases and becomes negative at $g \simeq 0.15$ signaling that the system has reached the BEC phase. Finally, the superconductive phase is energetically stable.

This qualitative behavior persists for moderate values of $\delta \mu$, but increasing $\delta \mu$ eventually a transition to the normal phase occurs. The nature of this transition we now discuss.

For any fixed value of the coupling constant $g$ we denote by $\delta \mu_{c}$ the largest chemical potential difference that the homogeneous superconductive phase can sustain. In other words for $\delta \mu>\delta \mu_{c}$ the system enters the normal phase. Clearly $\delta \mu_{c}$ depends on the coupling strength $g$. In the weak coupling regime $(g \rightarrow-\infty)$ the value of the critical polarization approaches the Clogston limit [4] $\delta \mu_{c} \simeq \Delta_{0} / \sqrt{2}$, where $\Delta_{0}$ is the value of the gap at $\delta \mu=0$. The phase transition between the superconductive and the normal phase is first order. With increasing values of $g$ the value of $\delta \mu_{c}$ increases and the phase transition remains first order for values of the coupling smaller of $\simeq 0.13$.

For values of the coupling in the range $0.13 \lesssim g \lesssim 0.175$ the gap equation has three non trivial solutions in a range of values of $\delta \mu$ close to the critical mismatch. One of these solutions corresponds to a maximum of the free-energy, 
the other two to local minima. The minima are favored for different values of $\delta \mu$. At small values of $\delta \mu$ the favored state is the one with $\Delta=\Delta_{0}$. For values of $\delta \mu$ larger than a critical value the favored state is the second one, with $\Delta<\Delta_{0}$. The transition between these two states is first order. We remark that such behavior of the free-energy takes place only in the range of the coupling $0.13 \lesssim g \lesssim 0.175$. For values smaller than $\sim 0.13$ there is one phase transition from the homogeneous to the normal phase. For values of $g$ large than $\sim 0.175$ one of the minima of the free-energy disappears and, increasing $\delta \mu$, one finds a second order phase transition from the normal phase to the unpaired phase.

In order to clarify the behavior in the above-mentioned range of $g$, we plot in Fig. \ the free-energy difference $F-F_{0}\left(F_{0}\right.$ the value at $\left.\Delta=0\right)$ as a function of $\Delta$ for various values of $\delta \mu$ at $g=0.135$, i.e. inside the interval $[0.13,0.175]$. For each value of $\Delta$, the value of $\mu$ is determined by the equation $\partial F / \partial \mu=0$, corresponding to Eq. (92). We notice that, since the total number density is fixed, the average chemical potentials of the broken $(\Delta \neq 0)$ and normal $(\Delta=0)$ phases are in general different. For $\delta \mu=0.936 \epsilon_{F}$ the free-energy has a global minimum at $\Delta=\Delta_{0} \simeq 0.95 \epsilon_{F}$ and a local minimum at $\Delta \simeq 0.75 \epsilon_{F} ;$ at $\delta \mu=0.940 \epsilon_{F}$ the two minima are almost degenerate, and the values of the gap at the local minima are $\Delta=\Delta_{0}$ and $\Delta \simeq 0.625 \epsilon_{F}$; finally for $\delta \mu=0.942 \epsilon_{F}$ the former local minimum becomes the global one (and vice-versa), and the gap at the global minimum is $\Delta \simeq 0.6 \epsilon_{F}$. For higher values of $\delta \mu$ the value of the gap decreases monotonically and for $\delta \mu=\delta \mu_{c} \sim 0.955 \epsilon_{F}$ the system has a second order phase transition to the normal phase.

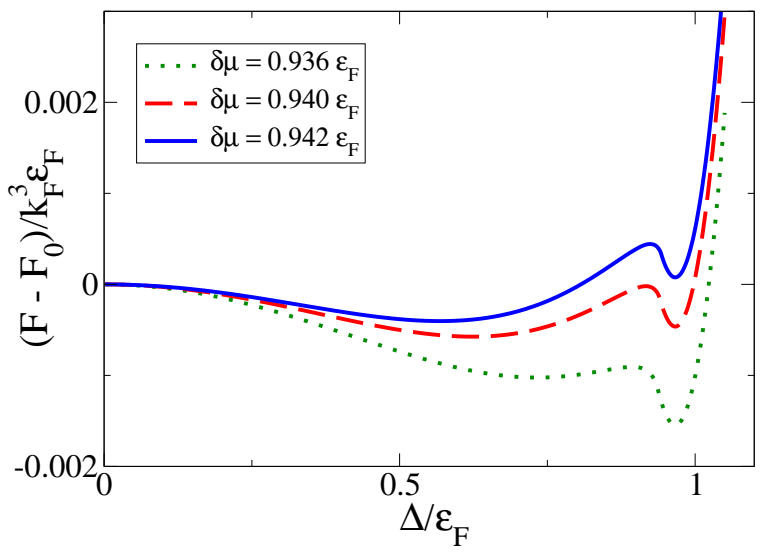

FIG. 1: Free energy difference $F-F_{0}$ as a function of $\Delta$ for various values of $\delta \mu$ at $g=0.135$.

The dependence on $g$ of the order of the phase transitions is shown on the left panel in Fig. 2 by three representative values of the dimensionless coupling constant, one inside the interval [0.13, 0.175], another one on the left, and a third one on the right of the interval. The lowest curve refers to $g=-0.1$. We have not considered here the possibility of inhomogeneous superconductivity and therefore we have a first order phase transition from the superconductive to the normal state. It occurs at $\delta \mu \simeq 0.79 \Delta_{0}$. For $0.79 \lesssim \delta \mu / \Delta_{0} \leq 1$ the superconductive phase becomes metastable and is shown as a dotted line. The highest curve is computed at $g=+0.2$ : for this value the transition from the superconductive to the normal phase is second order. The intermediate curve is obtained at $g=+0.135$ and shows, in agreement with the results of Fig. [1 two phase transitions: a first order phase transition from the value 0.95 to the value 0.65 of the gap parameter, and a second order phase transition to the normal phase. The values corresponding to the metastable phases are depicted as dotted curves. An enlarged picture of this case is in the inset. On the right panel in Fig. 2 we show the behavior of the average chemical potential $\mu$ as a function of $\delta \mu$, for the same values of the dimensionless coupling constant $g$. In the figure, the upper curve (green online) represents the average chemical potential in the normal phase The inset refers again to $g=0.135$.

It is also worth mentioning that the first order phase transition between the two minima of the free-energy corresponds to a phase transition between a gapped and gapless phase. The gapless phase is characterized by having one zero in the quasiparticle dispersion law at one sphere in momentum space. Had the dispersion laws two zeros then the system could live in the Breached Pairing phase [9, 10, but this possibility is not realized in this model at least within the present approximations. To illustrate this point we have reported in Figure 3 the results for $\mu / \Delta$ vs. $\delta \mu / \Delta$ as lines (green online) labeled with various values of $g$. Since for some values of $g$ there are first order phase transitions, some regions of this diagram are never reached by stable physical states, which is why in some cases the lines are interrupted. Such regions are above the thick full (red online) line, which has been determined comparing the energies of the various phases, and have been labeled with the letters $\mathbf{A}, \mathbf{B}$ and $\mathbf{C}$. The regions labeled as $\mathbf{A}$ and 

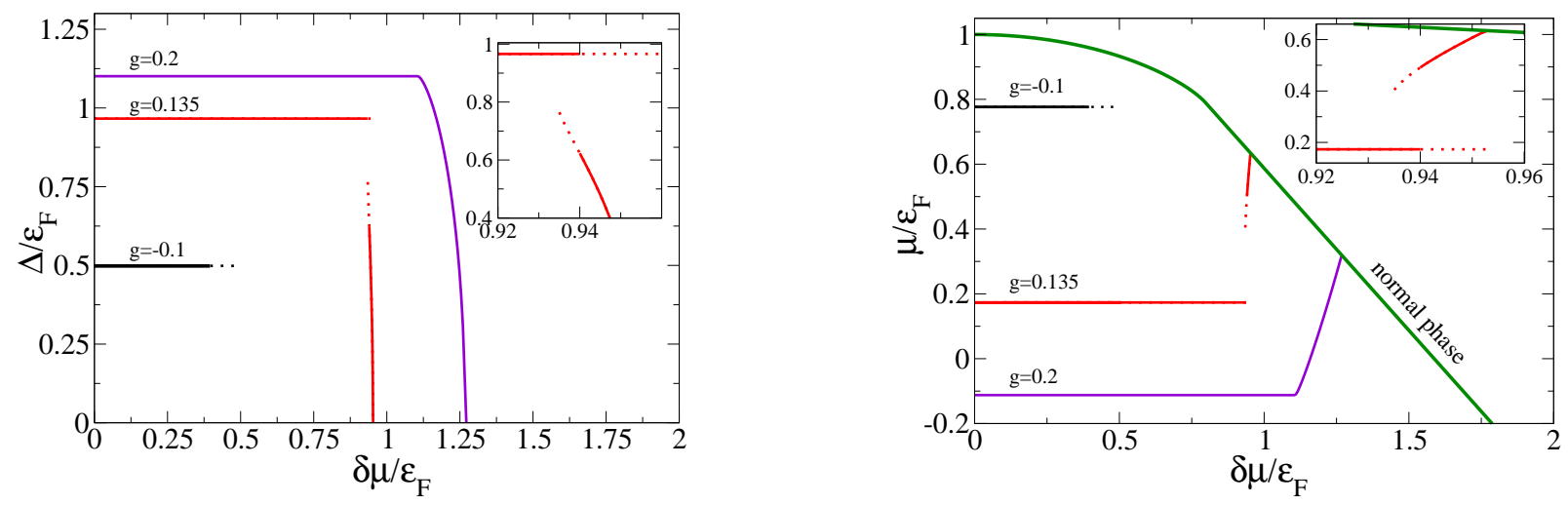

FIG. 2: On the left: The gap $\Delta / \epsilon_{F}$ vs. $\delta \mu / \epsilon_{F}$ for three values of the dimensionless coupling $g$. From top to bottom the lines refer to $g=0.2$ (purple online), $g=0.135$ (red online) and $g=-0.1$ (black online). The $g=0.2$ curve shows a second order phase transition to the normal phase at $\delta \mu / \epsilon_{F} \simeq 1.27$. The $g=-0.1$ curve shows a first-order transition to the normal phase at $\delta \mu \simeq 0.39 \epsilon_{F}$. The intermediate curve $(g=0.135)$, shown in more detail in the inset, shows the existence of two phase transitions. One phase transition is first-order. It leads to a superconductive phase with a different, smaller, value of the gap. The second transition leads smoothly to the normal phase. On the right: $\mu / \epsilon_{F}$ vs. $\delta \mu / \epsilon_{F}$ for the same three values of the dimensionless coupling $g$. The continuous upper curve (green online) refers to the normal phase $(g \rightarrow-\infty)$. The other three curves from bottom to top refer to $g=0.2$ (purple online), $g=0.135$ (red online) and $g=-0.1$ (black online). The inset represents an enlargement of the curve at $g=0.135$. In both panels the dotted parts of the $g=0.135$ and of the $g=-0.1$ lines correspond to metastable states.

C correspond to metastable points that are local minima of the free-energy. For $g=0.135$ they were reported in the insets of Fig. 2 as dotted points in the upper curve and lower curve respectively. The points in region labeled as $\mathbf{B}$ correspond to unstable BP points that are maxima of the free-energy. The remaining parts of the diagram correspond to allowed regions. The white area corresponds to the stable gapped phase and the shadow area (yellow online), with the exclusion of the region $\mathbf{C}$, to the stable gapless superconductive phase. In the shadow region $\delta \mu>\sqrt{\mu^{2}+\Delta^{2}}$ there are gapless excitations at one sphere in momentum space. All the regions meet at the point $\mathbf{P}$, on the line corresponding to $g=0.175$. The meaning of this point will be clarified below.

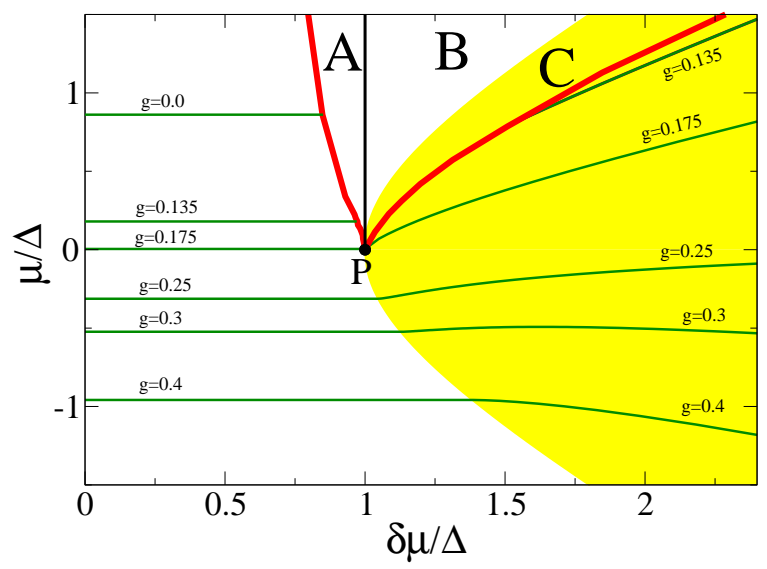

FIG. 3: Full (green online) lines are simultaneous solutions of the gap and number equations for different values of the coupling constant and of the mismatch between the Fermi spheres. The regions above the full (red online) line labeled with $\mathbf{A}$, B and $\mathbf{C}$ correspond to phases where no physical solutions of the gap and number equations have been found. The phase in region $\mathbf{B}$, the Breached Pairing phase, corresponds to unstable solutions. Regions $\mathbf{A}$ and $\mathbf{C}$ correspond to metastable phases. The shadow (yellow online) region, with the exclusion of the region $\mathbf{C}$, corresponds to the region with stable gapless solutions at one sphere in momentum space. The remaining part of the diagram corresponds to stable gapped solutions. 


\section{LOFF PHASE}

For values of $\delta \mu$ larger than $\delta \mu_{c}$ it can be energetically convenient for the fermionic system to form Cooper pairs with non-zero total momentum [6]. In the following we will consider a simple non homogeneous LOFF condensate characterized by a single plane wave

$$
<\psi_{\alpha}(x) \psi_{\beta}(x)>=\Delta \epsilon_{\alpha \beta} e^{i 2 \mathbf{q} \cdot \mathbf{x}},
$$

where $\mathbf{2 q}$ is the total momentum of the pair. More complicated patterns, such as those arising by more plane waves, may lead to states with a lower free-energy, but for our illustrative purposes the ansatz (10) is sufficient.

In the LOFF phase the quasiparticle dispersion laws are given by

$$
\epsilon_{1}(\mathbf{q})=+\delta \mu-\frac{\mathbf{q} \cdot \mathbf{p}}{m}+\sqrt{\xi(q)^{2}+\Delta^{2}}, \quad \epsilon_{2}(\mathbf{q})=-\delta \mu+\frac{\mathbf{q} \cdot \mathbf{p}}{m}+\sqrt{\xi(q)^{2}+\Delta^{2}},
$$

where we have defined $\xi(q)=\frac{p^{2}+q^{2}}{2 m}-\mu$. The free-energy for the LOFF phase can be written as

$$
\Omega(q)=\frac{\Delta^{2}}{G}-\frac{1}{2} \int \frac{d^{3} p}{(2 \pi)^{3}}\left[\left|\epsilon_{1}(\mathbf{q})\right|+\left|\epsilon_{2}(\mathbf{q})\right|-2 \xi(q)\right]
$$

and the integral is regulated as in the homogeneous case, i.e. by employing the S-wave scattering length defined in Eq.(6). In addition to the gap and number Equations (8),(9) one has to consider the equation

$$
\frac{\partial \Omega}{\partial q}=0
$$

which determines the modulus of $\mathbf{q}$; the direction of $\mathbf{q}$ is spontaneously determined by the system. Therefore in the non-homogeneous phase one has to solve a system of three coupled equations: the number equation, the gap equation and Eq. 113) for $\mu, \Delta$ and $q$ as a function of $g$ and $\delta \mu$ and to look for minima of the free-energy $F=\Omega+\mu n$.

From general arguments 23] one knows that the one-wave LOFF phase is energetically favored for values of the mismatch $\delta \mu$ in some interval: $\delta \mu_{1}<\delta \mu<\delta \mu_{2}$. Let us define the amplitude of the LOFF window as $\delta \mu_{21}=\delta \mu_{2}-\delta \mu_{1}$. Since the free-energy in the one-wave LOFF phase differs only slightly from the value in the normal phase, it turns out that $\delta \mu_{1} \simeq \delta \mu_{c}$. In the previous Section we have found that $\delta \mu_{c}$ is an increasing function of $g$. Comparing the free-energy of the LOFF phase with the free-energy of the normal phase we obtain that also $\delta \mu_{2}$ is an increasing function of $g$ but $\delta \mu_{21}$ turns out to be a non monotonic function of the coupling. In the weak coupling regime we recover the well known result $\delta \mu_{2} \simeq 0.754 \Delta_{0}$ and therefore $\delta \mu_{21}=(0.754-0.707) \Delta_{0}=0.047 \Delta_{0}$ is an increasing function of $g$. However in the intermediate coupling regime $\delta \mu_{1}$ grows faster than $\delta \mu_{2}$ and the amplitude of the LOFF window, depicted in Fig 4 reaches its maximum amplitude at $g \simeq-0.1$ and then begins to shrink; for $g \simeq 0.05$ the LOFF phase disappears.

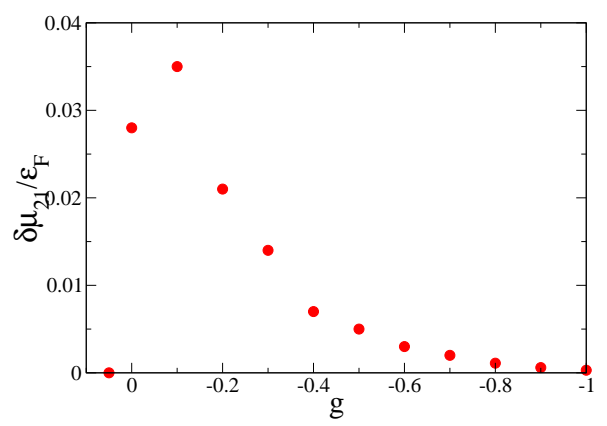

FIG. 4: Amplitude of the LOFF window $\delta \mu_{21}=\delta \mu_{2}-\delta \mu_{1}$ as a function of the coupling $g$. The LOFF window has its maximum amplitude for $g \simeq-0.1$ and shrinks to zero for $g>0.05$.

The largest value of the LOFF window is about $0.035 \epsilon_{F}$. However, as we have already mentioned, this value is a lower bound for $\delta \mu_{21}$ because more complicated crystalline structures might be able to sustain larger values of the mismatch between the Fermi surfaces. 
As a check of our results, we have determined the second order phase transition lines of the phase diagram by a Ginzburg-Landau (GL) expansion of the grand potential $\Omega$, both in the homogeneous and in the LOFF phase. The use of this approximation is justified because also in the strong coupling regime one has $\Delta / \delta \mu \rightarrow 0$ near the second order lines. Since we are interested to the second order transitions, it is enough to expand $\Omega$ up to the fourth order in $\Delta$, so the grand potential can be written as

$$
\Omega=\Omega_{0}+\frac{\alpha}{2} \Delta^{2}+\frac{\beta}{4} \Delta^{4}
$$

where $\Omega_{0}$ is the free gas contribution and the coefficients are given by

$$
\begin{gathered}
\alpha=\frac{2}{G}+T \sum_{n=-\infty}^{\infty} \int \frac{d^{3} p}{(2 \pi)^{3}} \frac{2}{\left(i \omega_{n}-\epsilon_{1}\right)\left(i \omega_{n}+\epsilon_{2}\right)}, \\
\beta=T \sum_{n=-\infty}^{\infty} \int \frac{d^{3} p}{(2 \pi)^{3}} \frac{2}{\left(i \omega_{n}-\epsilon_{1}\right)^{2}\left(i \omega_{n}+\epsilon_{2}\right)^{2}} .
\end{gathered}
$$

In Eqs. (15) the $\epsilon_{\sigma}$ are the dispersion laws of the quasi-particles,

$$
\epsilon_{1}=\frac{(\mathbf{p}+\mathbf{q})^{2}}{2 m}-\mu_{1}, \quad \epsilon_{2}=\frac{(\mathbf{p}-\mathbf{q})^{2}}{2 m}-\mu_{2}
$$

(the homogeneous case is studied by putting $\mathbf{q}=0$ in the above expressions). The divergence in the integral defining the coefficient $\alpha$ is cured, by the introduction of the S-wave scattering length, as discussed in Section Using the GL expansion we reproduce within a few percent the second order transition lines obtained by the numerical evaluation of the free-energy minima in the full theory .

\section{PHASE DIAGRAM}

We summarize our results in the phase diagram depicted in Fig. 5 In the following discussion of the phase diagram we will show that there is a correspondence between some regions and lines of the phase diagram and of the diagram depicted in Fig. [3]

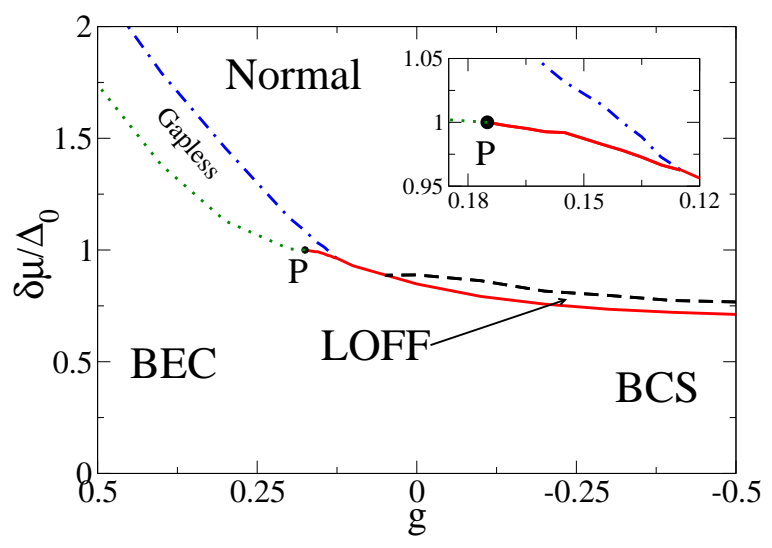

FIG. 5: Phase-diagram at $T=0$. The full line (red online) indicates the first order phase transition between the homogeneous gapped superfluid phase and the LOFF phase for $g \lesssim 0.05$ or the normal phase for $0.05 \lesssim g \lesssim 0.13$ or the gapless homogeneous superfluid phase for $0.13 \lesssim g \lesssim 0.175$. The dashed line (black online) indicates the second order phase transition between the LOFF phase and the normal phase. The dot-dashed line (blue online) indicates the second order phase transition between the homogeneous superconductive phase and the normal phase. The dotted (green online) line, which does not correspond to a phase transition, separates the homogeneous gapped phase from the homogeneous gapless phase. In the inset it is shown that the full line continues beyond the point where the dot-dashed line and the full line meet.

To begin with we describe the full line (red online). At values of $g \lesssim 0.05$ it indicates the first order phase transition between the homogeneous superconductive phase and the LOFF phase. For $0.05 \lesssim g \lesssim 0.13$ the full line indicates a 
first order phase transition from the superconductive phase to the normal phase. The regions corresponding to the LOFF phase and to the normal phase are not depicted in Fig. [3]

For $0.13 \lesssim g \lesssim 0.175$, the full line represents the first order phase transition between the superfluid gapped phase and the superfluid gapless phase (see the discussion in Section \in connection with Figs. \and 2). From the inset of Fig. 5 one can see that such a first order phase transition line terminates at the point $\mathrm{P}$, at $g \sim 0.175$. This point is depicted in Fig. 3 by the same letter. The full (red) line shown Fig. $\mathbf{5}$ corresponds to the full (red) line shown in Fig. 3 One can understand the different topologies of Figs. 5 and $\mathbf{3}$ in the following way. Since the regions A, B and $\mathbf{C}$ in Fig. [3 are not physical they cannot be reported in the phase diagram of Fig. 5 One can naively say that in the phase diagram the areas of regions $\mathbf{A}, \mathbf{B}$ and $\mathbf{C}$ have been shrunk to zero. Therefore once the two branches of the

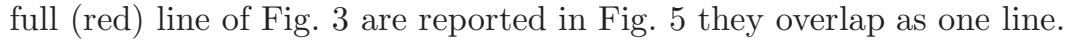

For larger values of $g \gtrsim 0.13$ we do not find a phase transition between the gapped and the gapless phase which are separated in Fig. 5 by the dotted (green online) line. Such a line corresponds to the onset of the shadow (yellow online) region of Fig. 3 for negative values of $\mu$. The shadow region (with the exception of the region $\mathbf{C}$ ) shown in Fig. 3 corresponds to the region between the dotted green line and the dot-dashed line ( blue online) of Fig. 5 The dot-dashed line represents the second order phase transition between the superfluid phase and the normal phase.

The phase transition between the LOFF phase and the normal phase (dashed red line) is of the second order. This result, already known in the weak coupling approximation [6], persists also for intermediate values of the dimensionless coupling constant, i.e. up to $g \sim+0.05$. For this value of $g$ and for $\delta \mu \sim 0.8 \epsilon_{F}$, the transition lines LOFF-BCS and LOFF-Normal phase cross. For values of the coupling constant larger than $g \sim 0.05$ the LOFF phase cannot be

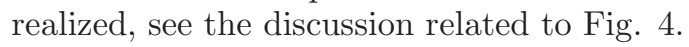

In conclusion we have analyzed in the mean field approximation the phase diagram of a fermion superfluid system comprising two unbalanced populations. We have worked at fixed total number density but arbitrary density difference, conversions between particles of different species are allowed thereof. As a consequence, at $T=0$, the phase diagram depends on two parameters, the dimensionless coupling constant and the mismatch $\delta \mu$ between the two Fermi spheres.

In view of possible applications to the study of the phase diagrams of systems composed of cold fermion atoms the present study should be considered as preliminary. As a matter of fact we have not included two effects. First, we have included only fermion self-interactions, without taking into account a confining potential. This development is postponed to future investigations. Second, we have not included fluctuations around the mean field solution. In the literature this is considered in general as a good approximation, at $T=0$ [31]. However, as discussed in [32], for values of the coupling smaller than some critical value (with our definitions, this corresponds to the region $|g|<0.1$ ) one enters a region where fluctuations may play a role. In general including fluctuations has the effect to enlarge the region of the ordered phase. This is revealed in the Quantum Monte Carlo computation of [32] by a gap in the dispersion law of the quasiparticle in a region where the normal phase should already be present. In other words the effect of the fluctuations would be to increase the transition line around $g \sim 0 \mathrm{in} \mathrm{Fig.} 5$ Therefore it is plausible that a more advanced study, would reveal in the region $|g|<0.1$ a more complex structure, more akin to the one proposed in [15]. We plan to come to this problem in the future.

\section{ACKNOWLEDGEMENT}

We would like to thank M. Alford, R. Casalbuoni, M. Ciminale, E. Gubankova, V. Laporta, K. Rajagopal, A. Schmitt and R. Sharma, for useful discussions. One of us (M.R.) would like to thank the Center for Theoretical Physics of MIT for kind hospitality. The work of MM has been supported by the "Bruno Rossi" fellowship program and by the U.S. Department of Energy (D.O.E.) under cooperative research agreement \#DE-FC02-94ER40818.

[1] E. Tiesinga, B.J. Verhaar abd H. T. C. Stoof, Phys. Rev. A 47, 4114 (1993); S. Inouye et al., Nature 392, 151 (1998); P. Courteille et al., Phys. Rev. Lett. 81, 69 (2004); J. L. Roberts et al., Phys. Rev. Lett. 81, 5109 (1998); E. Timmermans, P. Tommasini, M. Hussein and A. Kerman, Phys. Rep. 315, 199 (1999).

[2] D. M. Eagles, Phys. Rev. 186, 456 (1969); A. J. Leggett in Modern Trends in the theory of condensed matter, edited by A. Pekalski and J. Przystawa (Springer-Verlag, Berlin, 1980).

[3] C. A. R. Sá de Melo, M. Randeria, and J. R. Engelbrecht, Phys. Rev. Lett. 71, 3202 (1993).

[4] A.M. Clogston, Phys. Rev. Lett. 9, 266 (1962).

[5] G. Sarma, Phys. Chem. Solids 24, 1029 (1963).

[6] P. Fulde and R.A. Ferrell, Phys. Rev. 135, A550 (1964); A.I. Larkin and Y.N. Ovchinnikov, Sov. Phys. JETP 20, 762 (1965).

[7] H. Muther and A. Sedrakian, Phys. Rev. Lett. 88, 252503 (2002) arXiv:cond-mat/0202409. 
[8] P. F. Bedaque, H. Caldas and G. Rupak, Phys. Rev. Lett. 91, 247002 (2003) arXiv:cond-mat/0306694.

[9] W. V. Liu and F. Wilczek, Phys. Rev. Lett. 90 (2003) 047002 arXiv:cond-mat/0208052.

[10] E. Gubankova, W. V. Liu and F. Wilczek, Phys. Rev. Lett. 91 (2003) 032001 arXiv:hep-ph/0304016.

[11] M. M. Forbes, E. Gubankova, W. V. Liu and F. Wilczek, Phys. Rev. Lett. 94, 017001 (2005) arXiv:hep-ph/0405059.

[12] J. Carlson and S. Reddy, Phys. Rev. Lett. 95, 060401 (2005) arXiv:cond-mat/0503256.

[13] P. Castorina, M. Grasso, M. Oertel, M. Urban and D. Zappala, Phys. Rev. A 72 (2005) 025601 arXiv:cond-mat/0504391.

[14] C. H. Pao, S.-T. Wu, and S. K. Yip, arXiv:cond-mat/0506437

[15] D. T. Son and M. A. Stephanov, arXiv:cond-mat/0507586

[16] D. E. Sheehy and L. Radzihovsky, Phys. Rev. Lett. 96, 060401 (2006) arXiv:cond-mat/0508430.

[17] K. Yang, arXiv:cond-mat/0508484

[18] P. Pieri and G.C. Strinati, arXiv:cond-mat/0512354 J. Kinnunen, L.M. Jensen and P. Torma Phys. Rev. Lett. 96, 110403 (2006) arXiv:cond-mat/0512556; W. Yi and L.-M. Duan, Phys. Rev. A 73, 031604(R) (2006) arXiv:cond-mat/0601006; F. Chevy, arXiv:cond-mat/0601122 T.N. De Silva, E.J. Mueller, arXiv:cond-mat/0601314 M. Haque and H.T.C. Stoof, arXiv:cond-mat/0601321 C. H. Pao and S. K. Yip, arXiv:cond-mat/0604530

[19] Q. Chen, J. Stajic, S. Tan and K. Levin, Phys. Rep. 412,1 (2005).

[20] A. Bulgac, M. M. Forbes and A. Schwenk, arXiv:cond-mat/0602274

[21] K. Yang, arXiv:cond-mat/0603190

[22] E. Gubankova, A. Schmitt and F. Wilczek, arXiv:cond-mat/0603603

[23] R. Casalbuoni and G. Nardulli, Rev. Mod. Phys. 76, 263 (2004) arXiv:hep-ph/0305069

[24] M.W. Zwierlein, A. Schirotzek, C.H. Schunck, and W. Ketterle, Science 311, 492 (2006) arXiv:cond-mat/0511197.

[25] G.B. Partridge, W. Li, R.I. Kamar, Y.-a. Liao, and R.G. Hulet, Science 311, 503 (2006) arXiv:cond-mat/0511752 .

[26] M.W. Zwierlein and W. Ketterle, arXiv:cond-mat/0603489

[27] H.A. Radovan et al., Nature (London) 425, 51 (2003); A. Bianchi et al., Phys. Rev. Lett. 91, 187004 (2003).

[28] K. Kakuyanagi et al., Phys. Rev. Lett. 94, 047602 (2005).

[29] J. A. Bowers and K. Rajagopal, Phys. Rev. D 66, 065002 (2002) arXiv:hep-ph/0204079.

[30] I. Giannakis, D.f. Hou and H.C. Ren, Phys. Lett. B 631, 16 (2005) arXiv:hep-ph/0507306.

[31] J. R. Engelbrecht, M. Randeria and C. A. R. Sá de Melo, Phys. Rev. B 55, 015153 (1997).

[32] J. Carlson and S. Reddy, Phys. Rev. Lett. 95, 060401 (2005).

[33] For preliminary studies of asymmetric spin populations that include the effect of the trap see Ref. [18] 